\title{
Linx
}

Revue des linguistes de l'université Paris X Nanterre

64-65 | 2011

Les genres de discours vus par la grammaire

\section{Valeurs du présent français et genres de discours}

\section{Créola Thénault}

\section{(2) OpenEdition}

\section{Journals}

Édition électronique

URL : http://journals.openedition.org/linx/1410

DOI : $10.4000 / \operatorname{linx} .1410$

ISSN : 2118-9692

\section{Éditeur}

Presses universitaires de Paris Nanterre

\section{Édition imprimée}

Date de publication : 1 juillet 2011

Pagination : 155-172

ISSN : 0246-8743

\section{Référence électronique}

Créola Thénault, «Valeurs du présent français et genres de discours », Linx [En ligne], 64-65 | 2011, mis en ligne le 01 juillet 2014, consulté le 01 mai 2019. URL : http://journals.openedition.org/linx/1410 ; DOI : 10.4000/linx.1410 


\title{
Valeurs du présent français et genres de discours
}

\author{
Créola Thénault \\ Université Paris Ouest La Défense - CNRS MoDyCo
}

\section{Introduction}

La présente étude s'inscrit dans le programme épistémologique d'une linguistique néosaussurienne de l'interprétation et dans le champ pluridisciplinaire de la sémantique des textes et des sciences de la culture ${ }^{1}$. Cette linguistique se propose d'illustrer contrairement au point de vue strict adopté par le Cours de linguistique générale ${ }^{2}-$ la liaison nécessaire ou réquisit de complémentarité entre linguistique de discours et grammaire de la langue, que Saussure pose ainsi dans ses Écrits ${ }^{3}$ :

\footnotetext{
${ }^{1}$ Cf. Bouquet (2002 : 11-35).

2 «[...] la linguistique a pour unique et véritable objet la langue envisagée en elle-même et pour ellemême. » (Saussure, F. de, (1916) 2005 : 317). Comme le souligne Simon Bouquet dans une note (32) à son introduction dans un numéro de Linx $\left(n^{\circ} 56\right)$ "Il est significatif que la dernière phrase apocryphe du Cours (1916) soit empruntée à Bopp (1816), qui a précisément ouvert la voie, avec sa grammaire comparée, d'un siècle de linguistique logico grammaticale. Si Saussure, comparatiste, est disciple de Bopp en quelque manière, l'épistémologie programmatique saussurienne, contestant le paradigme de «la langue en elle même et pour elle même », s'oppose diamétralement à Bopp de ce point de vue : Le malentendu où tomba au début l'école fondée par F[ranø] Bopp fut de prêter aux langues un corps et une existence imaginaires en dehors des individus parlants. (Écrits de linguistique générale, p. 129)» (Bouquet, $2008: 13$, nous soulignons).

${ }^{3}$ Écrits de linguistique générale [S. Bouquet et R. Engler (éds.)], 2002. Abréviation : ÉLG.
} 
«Sémiologie $=$ morphologie, grammaire, syntaxe, synonymie, rhétorique, stylistique, lexicologie, etc., le tout étant inséparable ${ }^{4}$.

En partant de quelques principes définis dans le cadre de cette épistémologique générale ${ }^{5}$, je vais présenter l'esquisse d'une grammaire (ou sémiotique) de langue systématisant les différentes valeurs oppositives du temps (dit encore tiroir verbal) «présent » dans une perspective synchronique. Il s'agit d'une description notant (littéralisant) dans un système virtuel, des «traits de signifiés » locaux, représentés dans une arborescence sous la forme d'une algèbre différentielle. L'actualisation des «signifiés locaux » se fera, dans le cadre de cette analyse, par des «traits de signifiés» globaux ou génériques. Le genre ${ }^{6}$ ou simple "signifié générique» sera la notation oppositive d'une valeur différentielle ad hoc globale.

Dans cette optique, tout en empruntant largement leurs appellations classiques aux grammaires, l'analyse tâchera de montrer: 1) comment une différence de trait sémiotique suffit à décrire le «sens » du morphème présent en français; 2) et comment l'unification de la langue et de la parole (ou des genres de discours) réside dans l'écriture, pour une séquence discursive donnée, de « lois de corrélation » entre ces deux paliers linguistiques.

\section{Principes méthodologiques d'une linguistique néosaussurienne}

On peut et on doit considérer le présent linguistique comme étant de sa nature même - ainsi que le disait Saussure à propos de toute entité linguistique, et de la langue elle-même - un « signe complexe destitué d'une unité naturelle » :

Les éléments premiers sur lesquels portent l'activité et l'attention du linguiste sont non seulement d'une part des éléments complexes, qu'il est faux de vouloir simplifier, mais d'autre part des éléments destitués dans leur complexité d'une unité naturelle

\footnotetext{
${ }^{4}$ ÉLG : 45, soulignement de Saussure.

5 Épistémologie compatible en même temps avec le programme d'une linguistique des genres de Bakhtine et avec le projet d'une herméneutique matérielle de Schleiermacher, mais aussi avec la philosophie des jeux de langage de Wittgenstein (Bouquet, 2008a : 12-13).

${ }^{6}$ «Le mot genre, bien établi dans la tradition de la rhétorique et de la théorie littéraire, doit s'entendre ici dans une acception très générale : comme une appellation commode pour désigner un trait global de sens, dit encore signifié global, existant dans tout texte écrit ou oral. De fait, cette notion de «signifié global» correspond assez précisément à ce que Wittgenstein désigne comme des jeux de langage, tout autant qu'à ce que Schleiermacher (et, après lui, Bakhtine) nomme genre. À ces notions philosophiques font écho des objets conceptuels plus ou moins intuitifs, familiers à diverses approches d'analyse textuelle ou discursive, mais souffrant d'un certain déficit épistémologique: discours, type, mode, genre, champ générique, sous-genre, registre, domaine, niveau, isotopie, thème, etc. La révolution sémiotique saussurienne permet de regarder tous ces objets conceptuels $1^{\circ}$ comme se rapportant à des variétés de signifiés globaux, $2^{\circ}$ comme relevant d'une analyse différentielle. » (Bouquet, 2011c : 1, note 4.). Pour le genre comme fonction lire Bouquet, 2004c.

${ }^{7}$ ÉLG : 18, soulignement de Saussure.
} 
Poser que l'objet de notre analyse, le présent linguistique est un signe destitué d'une unité naturelle, revient à adopter pour celui-ci les mêmes principes que l'on pose pour la linguistique en général. Ces principes, que nous citons d'après Simon Bouquet $^{8}$, relevant d'une épistémologie dite " galiléenne » fondée sur la différentialité ${ }^{9}$ et formant système sont :

1.1. Le principe de légalité ;

1.2. Le principe d'empiricité ;

1.3. Le principe de sémioticité ;

1.4. Le principe méthodologique.

\subsection{Principe de légalité (= scientificité)}

La linguistique est une science énonçant des lois ; les lois qu'elle énonce doivent satisfaire aux critères généraux de scientificité : i) littéralisation; ii) formalisation; iii) réfutabilité ${ }^{10}$.

En tant qu'il s'attache à une telle épistémologie, l'objet de notre étude doit être un objet littéralisé qui entre dans des propositions formalisées configurées de telle sorte qu'elles puissent être réfutées.

\subsection{Principe d'empiricité}

La linguistique est une science empirique; son objet est un objet muni de coordonnées spatio-temporelles : ces coordonnées sont celles de l'événement psychique de l'interprétation.

L'objet observé est un fait d'esprit qui ne saurait exister en dehors du sujet parlant. L'observable, l'observatoire et l'observateur résident dans un même point: l'ESPRIT observable dans l'ESPRIT et par l'ESPRIT. L'objet présent de notre analyse linguistique est, à la fois un objet empirique et d'esprit.

\subsection{Principe de sémioticité}

À tous les niveaux de la linguistique les objets sont des signes. Le signe est conçu comme étant de sa nature à la fois : i) bifacial, synchronique et différentiel ; ii) régi par les trois principes : transversalité, compositionalité et herméneuticité.

\footnotetext{
${ }^{8}$ Cf. Bouquet (2012a : 22-30). Pour une exposition plus détaillée lire Bouquet (2012b).

${ }^{9}$ C'est surtout une manière d'inscrire notre objet d'étude dans le programme d'« une grammaire générale d'un type nouveau appréhendant ses objets sur la base du principe d'oppositivité intrasystémique (nommé encore négativité, différence, kénôme) et concevant ceux-ci comme les constituants d'une « mathesis linguistica » (cf. «Préface des éditeurs », ÉLG : 9-10).

${ }^{10}$ Nous ne citons ici que de manière sommaire ces critères galiléens. Pour une clarification de ces présupposés « galilléens », lire Milner (1989, Première partie) et Bouquet (1997 : 17-53).
} 
Le principe de sémioticité pose le « signe » comme seul objet de la linguistique. Le présent est un signe dont la valeur est strictement différentielle et dont l'interprétation est déterminée par le double principe de compositionalité et d'berménenticitée ${ }^{11}$.

\subsection{Principe méthodologique}

La méthode d'une linguistique de l'interprétation consiste à décrire le sens d'une séquence de langage par des lois corrélant le niveau sémiotique global (le signifié ou « genres de discours) et le niveau sémiotique local (le signifiant ou « grammaire de langue »). Ces niveaux linguistiques, littérarisés dans une algèbre différentielle grâce aux lois d'interprétation opposant la séquence analysée à une autre séquence homonyme, seront notés dans une écriture formelle. L'homonymie segmentale y joue le rôle de gage de la réfutabilité des lois différentielles.

L'objet de notre étude - satisfaisant aux critères de l'épistémologie " galiléenne » (littéralisation = la notation algébrique des valeurs différentielles du morphème présent, formalisation $=$ l'établissement des lois de corrélation utilisant cette littéralisation, réfutabilité $=$ le jugement de différentialité des lois de corrélation) - se laissera ainsi capter dans une écriture formelle.

\section{Grammaire de langue et genres de discours des valeurs du présent}

Pour certains, le présent serait même « un point aveugle », difficile à constituer en objet d'étude puisque difficile à comprendre ${ }^{12}$. Devant la difficulté que pose la signification du présent, on a essayé plusieurs définitions, plusieurs types d'explications : des explications psychologisantes ${ }^{13}$, des explications phonologisantes ${ }^{14}$, des explications énonciatives ${ }^{15}$, etc. ${ }^{16}$

Les grammaires actuelles oscillent entre plusieurs points de vue et optent tantôt pour l'un, tantôt pour l'autre. On reconnait alors pour le présent, ce temps à allure tentaculaire $e^{17}$, comme pour tout autre temps verbal, trois systèmes de valeurs indépendants: i) le système des valeurs temporelles proprement dites; ii) le système des valeurs

\footnotetext{
11 Le principe de sémioticité est posé comme transversal non seulement à l'analyse strictement compositionnelle des valeurs de langue mais aussi à l'analyse non strictement compositionnelle de la parole. À tous les niveaux analysables du langage on a des «signes différentiels » : au niveau de la langue ou local on a des "signes de langue»; au niveau de la parole ou global on a des «signes de parole». Le «signe de parole» interprète «le signe de langue» (cf. principe herméneutique de détermination du local par le global de Schleiermacher, $1987:$ 67- 69).

12 Cf. Aristote (1862: 226).

13 Cf. Guillaume (1964 : 210-213) ; (1965 : 52-60) et Wagner et Pichon (1962, \ $397: 344)$.

${ }^{14}$ Cf. Jakobson (1963: 185).

${ }^{15}$ Cf. Damourette et Pichon (1970, t. 5, \1706 : 172) ; Benveniste (1986 : 238-245).

16 Pour une analyse plus détaillée de ces explications voir la critique de Touratier (1996).

${ }^{17}$ Cf. Imbs (1960 : 33).
} 
aspectuelles; iii) et le système des valeurs «stylistiques» ou littéraires. À cela s'ajoute un quatrième système iv) le système des valeurs modales ${ }^{18}$.

Toutefois, à notre connaissance : i) les grammaires d'hier ainsi que les grammaires d'aujourd'hui - parmi celles que nous avons pu consulter - ignorent l'organisation systémique des valeurs du présent ; ii) dans les grammaires anciennes et dans les grammaires modernes, malgré un effort remarquable de clarté et de simplicité, on note l'absence totale de tout système de formalisation (absence due peut-être au manque d'appareil méthodologique satisfaisant) ; iii) seule une grammaire différentielle, c'est-à-dire envisageant « des éléments destitués d'une unité naturelle » devrait se montrer, nous semble-t-il, apte à rendre compte de manière systémique des détails fins pour les valeurs du présent, grâce notamment à ses principes méthodologiques clairement définis. Dans cette optique, toute définition et/ou explication substantialistes étant hors de notre propos, nous allons présenter l'arborescence d'une grammaire sémiotique définissant les multiples valeurs oppositives que peut prendre le morphème présent en français.

\section{1. « Grammaire de langue »}

La présentation arborescente de cette grammaire reflète la nature oppositive des valeurs qu'elle entend définir pour plusieurs raisons : i) les traits de signifié (ou valeurs différentielles) présentés sont des traits binaires ${ }^{19}$ (ils s'opposent deux à deux ou

${ }^{18}$ Cf. Imbs (1960 : 20-53, 185-190 et 194-200).

${ }^{19}$ Par opposition aux autres modèles qui optent plutôt pour une arborescence ternaire, nous plaidons ici pour un binarisme se justifiant de la prise en compte maximale de la nouveauté bouleversante de la linguistique saussurienne. En effet, la nouveauté de la pensée saussurienne consiste en ceci : l'élaboration à la fois d'une épistémologie programmatique et d'une nouvelle métaphysique du langage. Cette métaphysique nouvelle n'est pas basée sur des unités, sur des entités ou identités corporelles. Elle n'a pas une conception référentielle du langage. Elle n'est pas non plus un modèle inférentiel de la communication. Ce dont il s'agit, c'est une métaphysique autre, une nowvelle ontologie basée sur le principe de différentialité. Cette ontologie est si nouvelle, qu'elle est indépendante de toute ontologie préétablie, parce-que sans cesse renouvelée. Saussure nous a ainsi appris à ne rien tenir pour évident. La seule évidence en linguistique n'étant qu'un fait d'esprit ; à la fois signe-idée, l'unité ici n'est qu'illusion, seule la différence est. Il ne semble pas que l'on en prenne toujours l'exacte mesure... Un jour, nous en sommes convaincue, cette innovation ontologique - sous une forme et à des fins qu'on ne peut imaginer-, retrouvera un usage plus fécond. Saussure non seulement a porté à son plus haut degré l'interprétation littéralisante de la linguistique, non seulement il s'est attaché à exposer clairement les principes et données d'une science, mais aussi et surtout il fonde en raison et en concepts cette science à venir : une science positive de faits d'esprit.

On peut ajouter à cela le fait que le binarisme (fondé sur la notion de "complémentarité » en théorie des ensemble) est l'expression la plus naturelle et la plus simple de l'interdépendance que Saussure nomme opposition, négativité, différentialité. Dans l'opposition ensembliste binaire A/B, un A peut être entièrement défini comme étant un non $B$ et un $B$ peut être entièrement défini comme étant un non A. En cela le binarisme de l'écriture est le garant de l'opposition/négativité/différentialité.

Ajoutons encore que cette opposition/négativité/différentialité, révolutionnaire certes en linguistique, n'est pas un scoop des ÉLG/LLG : elle est parfaitement posée dès le CLG (contrairement à la nature sémiotique et à l'inséparabilité langue/parole). 
autrement-dit, toute valeur qui sera numérotée « 1 » renvoie à la négation réciproque d'une valeur « 2 » de même niveau); ii) ces traits sont ordonnés de telle manière que chaque trait note une subdivision binaire du trait qui le domine) ; iii) les compositions de traits correspondant aux branches terminales de l'arborescence (en gras) sont celles actualisées par le morphème ; iv) chaque composition terminale de traits est finement décrite grâce à la possibilité des énoncés homonymes dont ces traits permettent de différencier le sens.

\subsubsection{Divisions de l'arborescence ${ }^{20}$}

La ramification tentaculaire que peut prendre notre arborescence des valeurs du présent, nous oblige à scinder celle-ci (par souci de lisibilité de notre numérotation binaire des traits distinctifs) en 4 parties ou divisions pouvant comporter plusieurs subdivisions. Ces divisions, sont désignées par des chiffres romains :

I. Division initiale : valeur sémiotique vs valeur non sémiotique

II. Division de la valeur déictique extratextuelle

III. Division de la valeur déictique intratextuelle

IV. Division de la valeur « symbolique »

L'étiquetage des traits grammaticaux morphémiques selon ces divisions, et selon leurs subdivisions est présenté dans la section qui suit.

\subsection{2. Étiquetage de traits grammaticaux morphémiques}

\section{Division initiale : valeur sémiotique vs valeur non sémiotique}

La division supérieure de la valeur sémiotique [+SÉM] s'opposant à la valeur non sémiotique [-SÉM], présente deux traits différentiels, complémentaires : le trait [+IDX] (valeur indexicale ou temporelle); et le trait [-IDX] (valeur non indexicale, non temporelle ou symbolique). Le trait [+IDX] se subdivise en deux traits oppositifs notés :

- $\quad[+\mathrm{EXT}]$ trait d'indexicalité extratextuelle, subsumant les valeurs dites classiquement de déixis temporelle;

- $\quad$ [-EXT] trait d'indexicalité intratextuelle, subsumant les valeurs dites classiquement de d'anaphore et coréférence (ou suiréférentialité) temporelles.

\footnotetext{
${ }^{20}$ La description de notre arborescence reprend l'intégralité des divisions de l'arborescence proposée par Simon Bouquet (2004b) pour les «pronoms personnels", divisions reprises en 2008b pour le «morphème on ». Par ailleurs, nous considérons le pronom et le verbe comme un seul domaine, ou si l'on veut un plexus de morphèmes : mode/temps/ et personne à la fois, appartenant syntaxiquement à l'inflexion (INFL) de la phrase et non au verbe. Cette valeur de signifié local, valeur dominant donc la phrase entière, se trouve dans un rapport très intime avec le signifié global (dont il est parfois significativement désigné par une valeur homonyme: "narration", "vérité générale», etc.). Cela montre bien la détermination du local par le global et l'origine véritable de la grammaire dans la parole.
} 
Schématiquement, on peut noter ces traits binaires dans l'arborescence (I) qui suit :

\section{Arborescence I :}

1. : +SÉM (valeur sémiotique «pleine »)

1.1. : +IDX

1.1.1. : +IDX/+EXT (déixis temporelle) [voir branche II.]

1.1.2. : +IDX/-EXT (suiréférence et anaphore temporelles) [voir branche III.]

1.2. : -IDX (valeur symbolique, atemporelle) [voir branche IV.]

2. : -SÉM (valeur non sémiotique « vide », pour les tours figés : présent fige ${ }^{21}$ )

$>$ Le présent des « expressions figées ». ${ }^{22}$

Dans les locutions interrogatives est-ce que, qui est-ce qui, le verbe est au présent dit présent des expressions figées. Il en est de même dans les expressions : c'est-ä-dire, c'est pourquoi, c'est tout juste, etc.

\section{Divisions de la valeur déictique extratextuelle ([IDX/+EXT])}

Dans la valeur dite "temporelle » ${ }^{23}$, ou de chronologie réelle, l'action est considérée comme se réalisant dans son actualité effective, contemporaine, plus ou moins simultanée de l'acte de la parole (d'où le nom de valeurs actuelles des grammaires) ou au contraire, se réalisant dans un temps virtuel, inactuel, postérieur ou antérieur à

${ }^{21}$ Il ne s'agit pas d'un verbe !

22 Si la définition qu'Aristote donne du présent en tant que " point indivisible », point fixe, immobile, identique et immuable est fort ancienne (cf. Physique, 1862 : 260) en revanche, l'étiquette de la valeur du présent dit « figé » est relativement récente dans les grammaires. On la trouve à partir du XIX e siècle, par exemple chez Boniface Alexandre sous le nom de "présent dans des formules 'expressions' précises» (cf. Grammaire française méthodique et raisonnée, 1883 : 280). On la trouve aussi chez Chevalier, Blanche-Benveniste, Arrivé, Peytard qui utilisent la dénomination de "présent des expressions figées » (cf. Grammaire Larousse du français contemporain, 1964 : 338). Dans la grammaire historique de Nyrop Kristoffer on retrouve cette valeur sous l'étiquette "présent 'figé' » (cf. Grammaire historique de la langue française, 1979 : 283). Enfin, Maurice Grevisse parle également de "présent figé» (cf. Le bon usage, 14e édition, 2007 : 1091). Les auteurs ne sont pas d'accord sur le statut qu'il faut accorder au dit «figement»: d'une part, on considère que le figement est total ou absolu (le présent ne pouvant dans ce cas être remplacé par aucune autre forme verbale); d'autre part, on considère que le figement n'est pas complet (et dans ce cas au contraire du précédent, on pourrait faire varier le temps du verbe). Plus généralement, on constate qu'il y a dans la langue moderne des locutions verbales figées qui gardent ordinairement le présent, même dans le cas où une syntaxe rigoureuse demanderait un temps passé et/ou futur. Si pour plusieurs d'entre elles, le figement remonte assez haut; en revanche pour d'autres, ce figement ne s'est pas encore tout à fait accompli. À cet égard on pourrait dire que le présent n'est pas encore figé, il est en voie de figement. C'est un temps qui s'arrête, suspend et statufie son vol, comme dans ce vers bien connu de Baudelaire : Le soleil s'est noyé dans son sang qui se fige. (cf. « Harmonie du soir » in Les Fleurs du mal, Paris, $2000: 67)$.

${ }^{23}$ Le présent ne peut être dit «temporel» (présent, passé, futur) que sur une base d'indexicalité ; partant, une caractéristique essentielle du présent dit «a-temporel » est le fait qu'il est désindexicalisé. Le test de ces valeurs «temporelles» (les opposant aux valeurs +IDX/-EXT et aux valeurs -IDX) est la possibilité des manipulations dans lesquelles le présent commute avec un passé ou un futur. 
l'acte de parole (valeurs non actuelles). Le trait d'indexicalité extratextuelle ([+EXT]), subdivision de la valeur indexicale ([+IDX]), se divise ainsi en deux traits que l'on note :

$-[+\mathrm{ACTU}]$, valeurs actuelles;

$-[-\mathrm{ACTU}]$, valeurs non-actuelles.

Les traits de la valeur déictique extratextuelle se laissent noter différentiellement dans l'arborescence (II) qui suit :

\section{Arborescence II :}

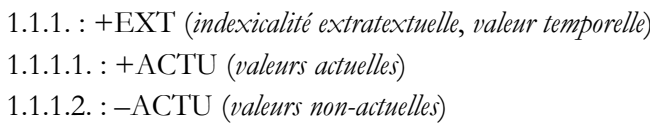

Subdivisions de la valeur 1.1.1.1 [+SÉM/+IDX/+EXT/+ACTU] de l'arborescence II

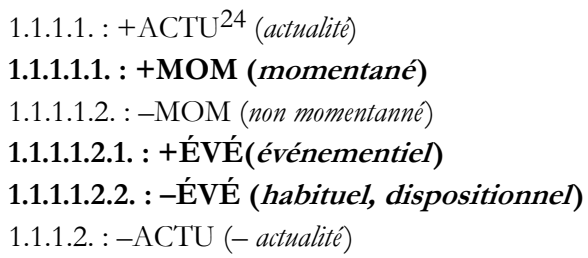

Les valeurs actuelles (Ex. (1) à (7) ci-dessous) couvrent une valeur momentanée ([+MOM]), de simultanéité stricte du procès avec l'acte de parole (présent actuel dans l'Ex. (1) et démonstratif en (2)), d'où le nom de présent simple, momentané, actuel, instantané, vrai, ponctuel ou non duratif que l'on rencontre dans les grammaires. Cette valeur s'oppose à la valeur non momentanée $([-\mathrm{MOM}])$ où au contraire, la durée désignée dépasse, déborde celle du moment de la parole comme le prouve le nom de présent étendu, élargi ou duratif que les grammaires lui reconnaissent. La durée qui déborde le temps de la communication du locuteur peut être plus ou moins symétrique du côté du passé et du futur (comme dans l'Ex. (4)), ou au contraire elle peut être asymétrique (comme dans l'Ex. (3)). Par opposition à ce présent événementiel ([+ÉVÉ]) ou occasionnel le présent non événementiel ([-ÉVÉ]) désigne une action qui prend toujours appui sur un procès actuel pour marquer une action habituelle, qui se répète (comme dans l'Ex. (7), et comme dans (5) et (6) une disposition plus ou moins permanente. Nous pouvons trouver ce présent dans les grammaires sous le nom de présent courant, dispositionnel, fréquentatif, habituel, répétitif, etc.
(1) Je suis là.
(2) Je prends un cuf, je le casse.
(3) Je suis là depuis/pour une semaine.
(4) L'eau est froide ce matin.
(5) Je suis là pour toi.
(6) Je suis là pour toujours.
(7) Elle ne réfléchit jamais.

${ }^{24}$ Valeur sémiotique d'indexicalité temporelle non passée et non future. 
Les traits différentiels de non actualité, se laissent capter dans l'écriture suivante :

Subdivisions de la valeur 1.1.1.2.[+SÉM/+IDX/+EXT/-ACTU] de l'arborescence II

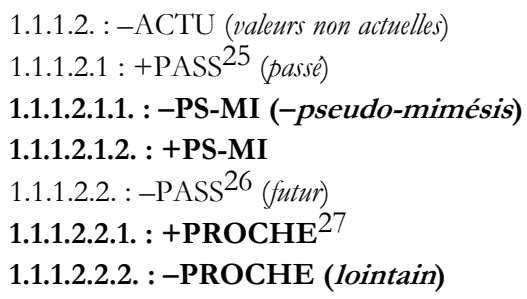

Les valeurs non actuelles (Ex. (8) à (13) ci-dessous), par opposition aux valeurs actuelles, se trouvent dans des énoncés où le temps est interprété comme étant soit antérieur à l'acte de parole (valeurs de passé non mimétique ([-PS-MI] comme dans l'Ex. (8)) vs de pseudo-mimésis ([+PS-MT]) ou pseudo-narratif (présent à valeur de passé séquentie ${ }^{8}$, présent «aoristique» selon Culioli comme dans (9)); soit au contraire postérieur (valeurs de futur, comme dans les Ex. (10), (11), (12), et (13)). Dans tous ces exemples, le présent est étendu, il déborde soit du côté du passé (plus ou moins récent) soit du côté du futur (plus ou moins proche [+PROCHE] ou lointain [-PROCHE]). Ce moment passé ou futur est identifié ici grâce aux compléments cotextuels.

(8) Je le quitte à l'instant

(9) Hier matin, je me lève, je prépare mon petit déjeuner, en prenant tout mon temps. Je vais dans la salle de bain, il n'y avait pas d'eau. J'attends, l'eau revient. J'étais en train de me savonner, quand il n'y a à nouveau plus d'eau. ${ }^{29}$

(10) J'arrive dans deux minutes.

(11) Dans trois mois, je ne travaille plus. 30

(12) Je prends ma retraite dans dix ans.

(13) En 2002, on passe à l'euro (dit en 2000).

\section{Divisions de la valeur déictique intratextuelle ([+IDX/-EXT])}

Le trait d'indexicalité intratextuelle ([-EXT]), subdivision de la valeur indexicale ([+IDX]), trait complémentaire à celui de l'indexicalité extratextuelle ([+EXT])

\footnotetext{
25 Valeur sémiotique d'indexicalité temporelle non actuelle et non future.

${ }^{26}$ Valeur sémiotique d'indexicalité temporelle non actuelle et non passée.

27 Les pseudo «futur proche» peuvent relever d'une durativité (J'arrive: je suis arrivant), d'une performativité (Je le fais le mois prochain: c'est promis); ou, comme dans le cas du présent à valeur de passé « séquentiel », d’une pseudo-mimésis (Voilà mon scénario: cette année, je travaille non stop, et l'année prochaine, je pars six mois en Australie).

${ }^{28}$ Hier, je sors, je rencontre Paul, il ne m'a même pas dit bonjour.

${ }^{29}$ Cf. Culioli (1990 : 136).

301 . Depuis trois mois, je ne travaille plus (actuel, étendu, passé) ; 2. Pendant trois mois, je ne travaille plus. (actuel, étendu/ passé/futur) ; 3. Dans trois mois je ne travaille plus (futur).
} 
subsume comme nous l'avons dit, les valeurs dites de suiréférentialite ${ }^{31}$ vs d'anaphore ${ }^{32}$. Ces traits se laissent noter ainsi :

- [+SUIRÉF], valeurs dites impérative, autonymique vs présent performatif, autosémique pouvant à son tour se subdiviser en une valeur de présent jussif légal ([+ LÉGAL] vs non légal ([-LÉGAL]);

- [-SUIRÉF], valeurs anaphoriques, de « concordance » ou dépendance syntaxique se divisant en deux traits oppositifs pouvant être notés: [+ANTÉ] (valeurs d’antécédence) vs [-ANTÉ] (valeurs de non-antécédence).

Les traits de la valeur déictique intratextuelle se laissent capter dans une notation différentielle dans l'arborescence (III) qui suit :

\section{Arborescence III :}

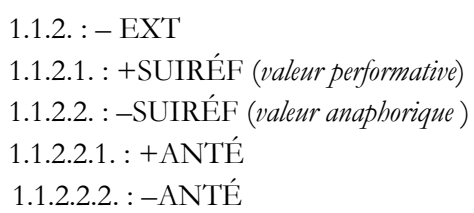

Subdivisions de la valeur 1.1.2.1. [+SÉM/+IDX/-EXT/+SUIRÉF] de l’arborescence III :

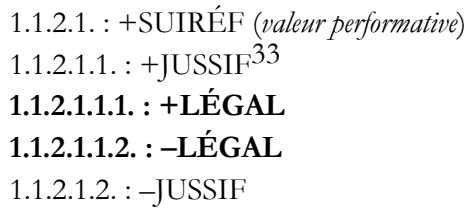

Les valeurs suiréférentielles, autophoriques ou de non dépendance syntaxique couvrent des valeurs performatives de présent jussif légal ${ }^{34}$ (Ex. (14) ci-dessous) vs non légal (Ex. (15) et (16)) s'opposant aux valeurs d'ordre non jussif (Ex. (17) et (18)).

(14) Les hommes naissent et demeurent libres et égaux en droits. ${ }^{35}$

(15) La Femme naît libre et demeure égale à l'bomme en droits. ${ }^{36}$

\footnotetext{
31 Valeur sémiotique d'indexicalité temporelle autophorique ou (auto-) référence du signe à lui-même.

32 Valeur sémiotique d'indexicalité temporelle anaphorique ou (alter-) référence du signe à un autre signe (« proforme » dans la terminologie de Chomsky, le fait qu'une «forme» reçoit son sens d'une autre « forme », dite classiquement « antécédent »).

${ }^{33}$ Le test de la valeur jussive de la suiréférence (l'opposant à la valeur non-jussive) est la possibilité de commutation du présent avec un futur.

34 Valeur « injonctive », « déontique », « directive » ou « normative », valeur d'« ordre » auquel on doit se plier; ici « légal », prévu dans le texte constitutionnel.

${ }^{35}$ Cf. La Constitution - Déclaration des Droits de l'Homme et du citoyen de 1789, Art. 1 ${ }^{\mathrm{er}}$.

${ }^{36}$ Cf. Olympe de Gouges, Article premier de la «Déclaration des droits de la femme et de la citoyenne », 1791.
} 

(16) Tu viens ! ${ }^{37}$
(17) Je t'aime.
(18) Je te hais.

Subdivisions de la valeur 1.1.2.2.1. : [+SÉM/+IDX/-EXT/-SUIRÉF/+ANTÉ] de

l'arborescence III :

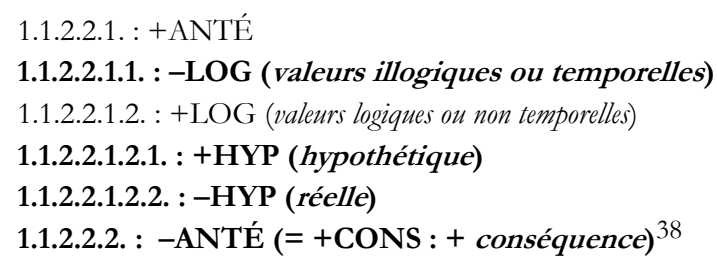

Les valeurs anaphoriques ${ }^{39}$ ou de subordination (dans les Ex. (19) à (39) cidessous) couvrent des valeurs d'antécédence ([+ANTÉ], Ex. (19) à (35)) s'opposant aux valeurs qui comme dans (36) à (39), ce sont des valeurs de non antécédence ([-ANTÉ]) ou de conséquence. Les valeurs d'antécédence distinguent en opposant deux autres valeurs : d'un côté, des valeurs d'antécédence temporelle, illogique ([-LOG ]), ne se trouvant pas dans un rapport de cause à effet (comme dans les Ex. (19) à (24) ci-dessous) et de l'autre côté des valeurs d'antécédence non temporelle, ou logique ([+LOG]) pouvant au contraire, se trouver dans un rapport de cause à effet (comme dans les Ex. (25) à (35)). Les valeurs d'antécédence temporelle peuvent se rapporter à une portion plus ou moins spécifique du temps, dans le passé Ex. (20) ou dans le futur Ex. (19) et comme dans l'Ex. (21) peuvent s'inscrire dans un intervalle de la durée non identifié avec précision. Enfin, les valeurs d'antécédence non temporelle s'opposent selon que l'on considère la subordonnée (avec ou sans connecteur) comme bypothétique ([+HYP]), potentielle, éventuelle ou irréelle, (Ex. (25) à (32)) ou au contraire non hypothétique ([-HYP]) ou réelle comme dans les énoncés (33) à (35) ci-dessous :

(19) Dès que tu es[Futur] là, on part.

(20) Depuis qu'il est là [Passé], il n'a pas desserré les dents.

(21) Depuis que je ne fume plus, je respire mieux.

(22) Nous nous détestons depuis que nous sommes toutes petites. ${ }^{40}$

(23) Si tu me le demandes [Futur], je le ferai.

(24) S'il pleut demain on ira se promener

(25) Si tu me le demandes [Présent], je le ferai.

(26) Tu me le demandes et je le ferai.

(27) Tu me le demandes et je le fais.

\footnotetext{
37 You shall do it!

38 Fais ça et tu verras!

39 Comme l'anaphore pronominale se rapporte à son GN antécédent, ici le morphème INFL-tense se réfère - via un connecteur, deux connecteurs ou un connecteur zéro - à l'INFL-tense d'une proposition subordonnante ou subordonnée.

${ }^{40}$ Illogique.
} 
(28) Sil quand on m'embête, je pars [Générique]

(29) Si on m'embête, je pars [Futur]

(30) Si on m'embête, je partirai.

(31) Tu me le demandes (et) je le ferai.

(32) Si je suis là, tu ne risques rien.

(33) Si (comme) tu me le demandes [Présent], je le ferai

(34) Tu me le demandes et je le fais.

(35) Si je suis là, tu ne risques rien.

(36) Un pas de plus et je tire.

(37) En jouant cour, tu gagnes à coup sûr.

(38) Tu me le demandes [Présent] et je le fais [Futur]

(39) Tu me le demandes [Cause], je le fais [Présent]

\section{Division de la valeur « symbolique » ([-IDX])}

$$
\text { 1.2. :-IDX (= désindexicalisê) }
$$

\subsection{1. : -MIM (valeur non mimétique ou « atemporelle») \\ 1.2.2. : + MIM (valeur mimétique ou « narrative »)}

Le trait de non indexicalité ([-IDX]) s'oppose en complétant le trait d'indexicalité $([+\mathrm{IDX}])$ de notre arborescence des valeurs du présent. Il note une valeur non déictique et non anaphorique a priori. Cette valeur dite ici symbolique, selon la terminologie de Peirce, couvre d'une part des valeurs dites "génériques» ou «atemporelles», non mimétiques ([-MIM], ce que les grammaires appellent par exemple présent des «vérités générales », « éthique », " gnomique », " proverbial », de «définition», des "raisonnements métaphysiques», etc. comme dans les exemples (40) et (41) ci-dessous) et d'autre part, des présents mimétiques ([+MIM]) ou de narration fictive. On rencontre ces valeurs dans tout récit fictif au présent d'évènements passés ou futurs, valeurs dites: canoniques «littéraires» ou «stylistiques ». Parmi celles-ci nous listons les valeurs suivantes : présent «tabellaire » (Ex. 42), «narratif» (Ex. 43) ou « historique » (Ex. 44), présent «prophétique » (Ex. 45), présent « pittoresque » (Ex. 46) ou de «description» (Ex. 47), de «didascalies» (Ex. 48) ou «scénique» (Ex. 49), etc.

(40) Les baleines sont des mammiferes.

(41) Les méchants sont toujours punis. ${ }^{41}$

(42) En 49 avant Jésus-Christ, César décide de franchir le Rubicon.

(43) Le comte Maréchal n'en peut plus. La charge maintenant l'écrase. ${ }^{42}$

(44) En 1789, le peuple de Paris prend la Bastille.

(45) Quelle Jérusalem nouvelle / Sort du fond du désert brillante de clarté / Et porte sur le front une marque immortelle ?43

(46) La Déroute apparut au soldat qui s'ément,

\footnotetext{
41 Valeur générique à différencier du présent dispositionnel : Les hommes sont toujours en retard. Ce qui distingue l'énoncé d'une vérité intemporelle de l'énoncé de faits habituels ou réguliers c'est que le premier ne peut se mettre au passé alors que le second le peut.

42 Duby, Guillaume le Maréchal, 1984 : 7.

${ }^{43}$ La prophétie de Joad chez Racine, Athalie, III, 1691 : 7.
} 
Et se tordant les bras, cria: sauve qui peut. ${ }^{44}$

Exemple (47) Tel qu'on voit un taureau qu'une guêpe en furie / A piqué les flancs aux dépens de sa vie / Le superbe animal, agité de tourments, / Exhale sa douleur en longs mugissements. ${ }^{45}$ Exemple (48) Maître Jacques pousse V alère jusqu'au fond du théâtre. ${ }^{46}$

Exemple (49) Le rideau s'ouvre sur une sorte de parade foraine. Au centre, une tenture devant laquelle, sur une petite estrade, se trowvent Hélicon et Coesonia. ${ }^{47}$

\section{3. «Genres de discours »}

\section{La langue n'est créée qu'en vue du discours ${ }^{48}$}

La langue ne se produit et ne se perçoit qu'à travers des genres ${ }^{49}$. Si le caractère fondamental de toute valeur du présent réside au niveau de la langue, comme on l'a constaté, dans une notation différentielle, de même la caractéristique essentielle de tout genre est qu'il relève du même principe baptisé différentialité ou inscrutabilité $d u$ sens: autrement dit, un genre n'existe que par opposition à un autre genre. Et, conformément au principe herméneutique de détermination du local par le global, une valeur de langue n'a d'existence qu'en tant que déterminée par une valeur générique. Un signifié local n'existe qu'actualisé par un signifié global: véritable origine de la forme de la langue, caractère fondamental de la parole

force active, origine véritable des phénomènes qui s'aperçoivent ensuite peu à peu dans l'autre moitié du langage. ${ }^{50}$

\subsection{Définition de la notion de genre}

Le «genre» est la notation oppositive d'une valeur générique (ou trait de signifié oppositif global). Celui-ci joue dans la constitution du sens d'un texte un double rôle: d'une part, c'est un élément dudit sens; d'autre part, il actualise les valeurs locales (signifiés grammaticaux) dudit texte ${ }^{51}$.

Si l'analyse sémiotique (ou grammaticale) de la langue ne fait que décrire des parties détachées de «sens », ne pouvant pas à elle seule rendre compte du processus de construction/compréhension du sens dans l'esprit d'un être humain, en revanche l'analyse sémantique, décrivant comment les valeurs virtuelles du présent sont actualisées par un genre, autrement-dit en utilisant le matériau grammatical, analyse le

\footnotetext{
${ }^{44}$ Hugo, Châtiments, V, v 131, 1853: 36.

45 Boileau, Lutrin, I, 1674 : 85.

46 Molière, cité par Wilmet, 2003 : 365.

47 Camus, Caligula, III, 1944 : 1.

48 ÉLG : 277.

49 Cf. Rastier (2001 : 273).

50 ÉLG : 273.

51 Ainsi défini, le concept de genre n'est ni le genre traditionnel, tel que l'on conçoit par exemple dans l'analyse littéraire, il n'est pas non plus celui de l'analyse du discours, ni celui des actes de langage.
} 
sens d'un segment de langage compris (interprété) par un sujet parlant. L'identification du genre se fait alors à partir de la double particularisation d'une variation linguistique et d'une différence pragmatique conçue sur la base d'un même principe d'opposition systémique. Nous inscrivant dans le cadre d'une sémantique (linguistique) des genres, notre analyse distingue et met en corrélation deux niveaux : le niveau de description grammaticale (ou sémiotique) répondant des «traits de signifié locaux» (que nous venons de décrire dans la section précédente) et le niveau de description sémantique répondant de l'actualisation de ces traits, par des "traits génériques globaux». Quelques exemples vont illustrer dans ce qui suit, la corrélation différentielle dans l'analyse du sens, d'une valeur générique et d'une valeur grammaticale du présent.

\subsection{Corrélations des valeurs du présent et genres de discours}

Le point d'entrée dans l'analyse néosaussurienne de l'interprétation pose deux énoncés dont la valeur du signifiant local est identique, et dont les valeurs du signifié temporel actualisées par des traits génériques - limitées à un simple «jugement de différence » - sont opposables. L'analyse qui suit se propose d'actualiser quatre traits oppositifs que l'on retrouve dans notre grammaire : présent à valeur plus ou moins actuelle; présent à valeur plus ou moins événementielle; présent à valeur plus ou moins passée ou future; présent à valeur d'antécédence plus ou moins temporelle (ou logique). Les corrélations différentielles (ci-dessous) de ces traits grammaticaux locaux avec des traits génériques globaux se laissent capter dans une écriture formelle (ci-dessous) :

Écriture formelle :

1. : [+ TrGé 1] (ou [-TrGé 2]) $=>$ [+ TrGr 1] (ou [-TrGr 2])

2. : $[+\operatorname{TrGé} 2]($ ou $[-\operatorname{TrGé} 1])=>[+\operatorname{TrGr} 2]($ ou $[-\operatorname{TrGr} 1])$

Corrélations différentielles :

3.2.1. $[+/-$ DIALOGUE $]=>$ [présent $+/-$ actuel $]$

3.2.2. $[+/-$ DISPOSITION OCCASIONNELLE] $=>$ [présent + /- événementiel]

3.2.3. [+/- DÉCISION PLANIFIÉE] $=>$ [présent $+/-$ passé]

3.2.4. $[+/-$ POÉSIE] $=>$ [présent d'antécédence $+/-$ temporelle $]$

3.2.1. $[+/-$ DIALOGUE $]=>$ [présent $+/-$ actuel]

Soit l'énoncé ou séquence de langage [É1] : «Je suis là», interprétable comme homonyme d'un autre signifiant ou signe textuel [É2] : "Je suis là». Le morphème temporel de cet énoncé sera interprété comme porteur d'un sens différent, selon que l'on considère dans des contextes ou genres différents, mais complémentaires. Ainsi notre morphème aura un Sens 1 dans un dialogue du couple $\mathrm{Mr}$ et Mme Dupont où Monsieur Dupont, cherchant (ne trouvant pas) sa femme dans la maison lui pose la question «Tu es où ? " pour la trouver, et celle-ci lui répond pour se faire localiser : «Je suis là!». Le trait générique de cet énoncé actualise le trait grammatical temporel, d'actualité momentanée, porté par le verbe être. De cette valeur, répond un test de commutation : il est impossible de remplacer le présent par le futur : on ne peut pas dire dans ce contexte * Je serai là. Au contraire, ce même morphème aura le Sens 2 
dans un autre contexte, opposé au premier par le fait que l'on n'est plus dans un dialogue mais dans un monologue philosophique : «Je pense donc je suis »; Descartes en train de philosopher sur l'existence, sur l'être en général se dit : "Je suis là ». Le trait grammatical d'omnitemporalité (ou atemporalité) porté par le verbe « être » dans cet énoncé est actualisé via le genre en question. Le test d'attestation de cette valeur constative non indexicale, valeur dite "présent de vérité générale » est la possibilité de mettre à côté du présent, le futur et le passé : J'ai été, je suis et je serai là.

On en déduit, pour le binôme [É1]/[É2] de Je suis là, la corrélation $[$ SéGé $]=>[$ SéGr $]$ suivante $:[+/-$ DIALOGUE $]=>$ [présent $+/-$ actuel $]$.

\subsection{2. $[+/-D I S P O S I T I O N$ OCCASIONNELLE] => [présent +/-événementiel $]$}

Soit le même segment phonologique, également dans un dialogue. Le présent du verbe « être » dans l'énoncé : "Je suis là » aura la valeur de présent + / - événementiel selon qu'il s'agit de répondre à une question pour dire ses disponibilités occasionnelles ou pas. Ainsi, on aura le Sens 1 dans le contexte suivant: dimanche en famille, Madame Dupont à Monsieur Dupont qui travaille au service de l'armée et qui est en permission pour une semaine lui demande : "Samedi prochain c'est l'anniversaire de notre petit fils, t'es encore là ? » et quand celui-ci répond : "Je suis là », on comprend bien qu'il est là, disponible de manière occasionnelle : il est là pour une semaine. Le trait grammatical porté par le verbe être au présent, a dans ce cas une valeur événementielle. Test de vérification de cette valeur : possibilité de remplacer le présent par le futur: on peut dire : je serai là. Et on aura le Sens 2 dans cet autre contexte : Madame Dupont, se sentant un peu seule dit à Monsieur Dupont qui bricole à sa voiture dans le garage depuis plusieurs semaines: "T'es là pour moi ou pas ? et quand celui-ci répond «Je suis là » (on entend bien je suis là pour toi », il lui répond en lui disant qu'il n'est pas là disponible pour elle comme ça, de manière occasionnelle mais au contraire, même si ... en réalité son bricolage a l'air de le captiver plus). Il semble clair que dans cette situation, et contrairement au cas précédent, le présent du verbe « être » est bien porteur du trait grammatical non événementiel. Pour le vérifier il suffit de constater que l'on ne peut pas ici remplacer le présent ni par le futur ni par le passé : * J'ai été là. * Je serai là.

On en déduit, pour le binôme [É1]/[É2] de Je suis là, la corrélation $[$ SéGé $]=>[$ SéGr $]$ suivante $:[+/-D I S P O S I T I O N$ OCCASIONNELLE $]=>[$ présent $+/$ événementiel ].

\subsection{3. $[+/-D E ́ C I S I O N$ PLANIFIÉE] $=>$ [présent $+/-$ passé]}

Soit maintenant la séquence de langage «Je ne travaille plus». Nous sommes toujours dans un dialogue où le présent du verbe «travailler» aura cette fois-ci une valeur de passé récent ou de futur plus ou moins proche selon que le genre de l'énoncé sera porteur du trait différentiel global +/- décision planifiée. On aura le Sens 1 dans ce contexte : Monsieur Dupont en train de discuter avec son meilleur ami en lui disant que dans trois mois il se passera quelque chose d'important dans sa vie ; à ce moment sa femme arrive et lui demande : «Il se passera quoi dans trois mois ? " et celui-ci lui répond : je ne travaille plus. Si la décision de ne plus travailler, est une décision planifiée, alors le trait grammatical porté par le verbe «travailler» au présent a la valeur de futur. 
Ce n'est pas le cas dans cet autre contexte où nous avons un sens différent : au travail, toujours Monsieur Dupont en sortant du bureau de son supérieur répond à son collègue un peu curieux, qui lui demande: «Alors, dis-moi pourquoi on t'a convoqué ? » et quand celui-ci lui répond en lui disant: «je ne travaille plus», on peut comprendre : j'ai été licencié à l'instant. Ici, il est clair qu'il ne s'agit plus d'une décision planifié, le verbe «travailler» semble alors porteur du trait grammatical que les grammaires appellent «passé récent».

On en déduit, pour le binôme [É1]/[É2] de Je ne travaille plus, la corrélation $[$ SéGé $]=>[$ SéGr $]$ suivante $:[+/-$ DÉCISION PLANIFIÉE $]=>$ [présent $+/-$ passé $]$.

\subsection{4. $[+/-$ POÉSIE] $]=>$ [présent d'antécédence $+/-$ temporelle]}

Soit l'énoncé « tu me le demandes, je le fais» auquel nous pouvons lui découvrir un Sens 1 dans ce contexte de conversation : Monsieur Dupont à Madame Dupont, qui est sur le point de sortir et qui lui demande un service : «Chéri, je peux te demander de laver la vaisselle pendant que je vais chez le coiffeur?» celui-ci accepte en répondant: (puisque) tu me le demandes, je le fais. Le trait grammatical du présent porté par le verbe «demander» est ici une valeur anaphorique d'antécédence temporelle.

En revanche, dans le genre poésie (cf. lessisters.free.fr/poemes.htm, poème d'amour: "Si tu me demandes de tuer tous ceux qui brisent ton cour je le ferai »), le même énoncé en plus de la valeur temporelle indexicale aura une valeur générique non déictique qui n'existe pas dans le genre conversation (au moment où nous l'interprétons et dans ce corpus).

On en déduit, pour le binôme [É1]/[É2] de Tu me le demandes, je le fais, la corrélation $[$ SéGé $]=>[$ SéGr $]$ suivante : $[+/-$ POÉSIE $]=>$ présent d'antécédence $+/$ - temporelle].

\section{Conclusion}

On constate que cette analyse ne produit aucun analogon substantiel du « sens ». À tous les niveaux de l'analyse, l'objet analysé est oppositif: les «traits de signifiés locaux ", les "traits de signifiés globaux», les «corrélations" elles-mêmes sont différentielles. Ainsi conçu, le seul objet de notre analyse reste la différence de sens entre deux énoncés, gagée sur l'homonymie textuelle du signifiant local.

Cette confrontation, de deux énoncés (« séquences textuelles» ou "corpus de signes textuels ») homonymes, a fait apparaitre clairement les traits grammaticaux pertinents pour la détermination de la corrélation entre «signifié générique » et «signifié grammatical " $^{52}$ du présent linguistique en français. La pratique d'une linguistique pure de la différence et de l'interprétation permet ainsi non seulement l'analyse des différences de sens du présent linguistique (cf. principe d'inscrutabilité du sens) mais aussi la plus fine description du sens de celui-ci. On entend par là le fait que l'unique analyse complète du « sens » d'un morphème ne saurait être qu'oppositive.

\footnotetext{
52 Cf. Pour plus de détails lire la Contribution de Bouquet S., 2004a : 28-40; et 2008 : 143-156 ; ainsi que l'article de Malrieu D., 2008 : 157-178.
} 
L'établissement des lois de corrélation différentielle des valeurs de langue avec des valeurs $d u$ discours du morphème verbal présent, satisfont enfin au réquisit de liaison nécessaire entre une linguistique de la parole et la grammaire d'une langue, le tout étant inséparable (cf. Saussure).

\section{BIBLIOGRAPHIE}

ARISTOTE, 1862, Physique ou leçons sur les principes de la nature, trad. par Job SAINT-HILAIRE, Paris, Ladrange.

BAkhtine, M., 1984, "Les genres du discours», in Esthétique de la création verbale, traduit du russe par Alfreda Aucouturier, Paris, Gallimard, p. 263-308.

Benveniste, É., 1986, «Les relations de temps dans le verbe français», in Problèmes de linguistique générale, T. I, Paris, Gallimard, p. 237-250.

Bouquet, S., 1997, Introduction à la lecture de Saussure, Paris, Payot.

—, 1998, «Linguistique textuelle, jeux de langage et sémantique du genre », in Langages, n²129, p. 112-123.

—, 2002, «De l'hexagramme cognitiviste à une sémiotique de l'interprétation », in F. Rastier et S. Bouquet (éds.), Une introduction aux sciences de la culture, p. 11-35.

—, 2004a, «Linguistique générale et linguistique des genres », in Langages, $\mathrm{n}^{\circ} 153$.

—, 2004b, «Sémiotique grammaticale et sémantique des (genres de) jeux de langage: les 'pronoms personnels' clitiques en français ", in Langages, $n^{\circ} 153$, p. 28-40.

—, 2004c, «Fonctions », in Niveau B2 pour le français, Paris, Didier, p. 63-119.

—, 2008a, «Introduction », in $\operatorname{Linx}, \mathrm{n}^{\circ}$ 56, p. 7-17.

—, 2008b, «Contribution à une linguistique néo-saussurienne des genres de la parole (1) : une grammaire sémiotique du morphème on », Linguistique des genres, in Linx, n 56, p. 143-156.

—, 2011b, «D’une épistémologie néosaussurienne de la linguistique à la question de l'universalité des droits de l'homme », in Rivista Italiana di Filosofia del Linguaggio, p. 52- 64.

—, 2011c, «Esquisse d'une sémantique de la déclaration universelle de droits de l'homme », in Texto! vol XVI, n³, p. 1-14.

—, 2012a, «Principes d'une linguistique de l'interprétation », in Langages, $n^{\circ} 185$, p. 21-33.

—, 2012b, «Triple articulation de la langue et articulation herméneutique du langage : quand De l'essence double du langage réinterprète les textes saussuriens ", in Arena Romanistica, sous presse.

CHOmsky, N., 1969, Structures syntaxiques, traduit de l'anglais par Michel Braudeau, Paris, Éditions du Seuil. 
Culioli, A., 1990, Pour une linguistique de l'énonciation, opérations et représentations, Tome I, Paris, Gap, Ophrys.

Damourette, J. et Pichon, É., 1970, Des mots à la pensée. Essai de grammaire de la langue française, Tome V, Paris, Éditions d'Arthrey.

Guillaume, G., 1965, Temps et Verbe. Théorie des aspects, des modes et des temps, Paris, H. Champion.

—, 1964, «De la double action séparative du présent dans la représentation française du temps », in Langage et sciences du langage, Québec et Paris, Presses de l'Université de Laval et A.-G. Nizet.

GrevisSE, M., 2007, 14ème édition, Bruxelles, De Boeck-Duculot.

IMBS, P., 1960, L'emploi des temps verbaux en français moderne; essai de grammaire descriptive, Paris, Klincksieck.

JAKOBSON, R., 1963, "Les embrayeurs, les catégories verbales et le verbe russe », in Essais de linguistique générale $I$ : les fondations du langage, traduit de l'anglais par Nicolas Ruwet, Paris, Éditions de Minuit, p. 176-196.

MALRIEU, D., 2008, «Contribution à une linguistique néo-saussurienne des genres de la parole (2): analyse des valeurs d'indexicalité interlocutoire de on selon les genres textuels », Linguistique des genres, in Linx, $\mathrm{n}^{\circ}$ 56, p. 179-195.

Milner, J.-Cl., 1989, Introduction à une science du langage, Paris, Éditions du Seuil.

NYROP, K., 1979, Grammaire historique de la langue française, Paris, A. Cotelle.

Rastier, F., 2001, Arts et sciences du texte, Paris, Presses Universitaires de France.

SAussure, F. de., 1916, Cours de linguistique générale [C. Bailly et A. Sechehaye (éds.) avec la collaboration de A. Riedlinger], Lausanne-Payot.

—, 2002, Écrits de linguistique générale [S. Bouquet et R. Engler (éds.)], Paris, Gallimard.

SChleiermacher, F., 1987, Herméneutique, traduit de l'allemand par Christian Berner, Cerf et Presses Universitaires de Lion.

Serbat, G., 1988, «Le prétendu 'présent' de l'indicatif : une forme non-déictique du verbe », in L'information grammaticale, $\mathrm{n}^{\circ} 38$, p. 32-35.

TOuratier, C., 1996, Le système verbal français, Paris, Armand Colin.

WAgneR, R. L. et PINCHON, J., 1962, Grammaire du français classique et moderne, Paris, Hachette.

WeInRICH, H., 1973, Le Temps : le récit et le commentaire, traduit de l'allemand par Michèle Lacoste, Paris, Éditions du Seuil.

Wilmet, M., 2003, Grammaire critique du français. Paris-Bruxelles, Hachette-Duculot. 